\title{
Peptide Oligomerization Memory Effects and Their Impact on the Physical Stability of the GLP-1 Agonist Liraglutide
}

Jameson R. Bothe ${ }^{+*}$, Alexandra Andrews ${ }^{\S}$, Katelyn J. Smith ${ }^{\S}$, Leo A. Joyce ${ }^{\dagger}$, Yogita Krishnamachari ${ }^{\S}$, Sandhya $\mathrm{Kashi}^{+}$

†Process Research and Development, Merck \& Co., Inc. Rahway, NJ, USA

"Pharmaceutical Sciences, Merck \& Co., Inc. Rahway, NJ, USA

\section{Supporting Information}

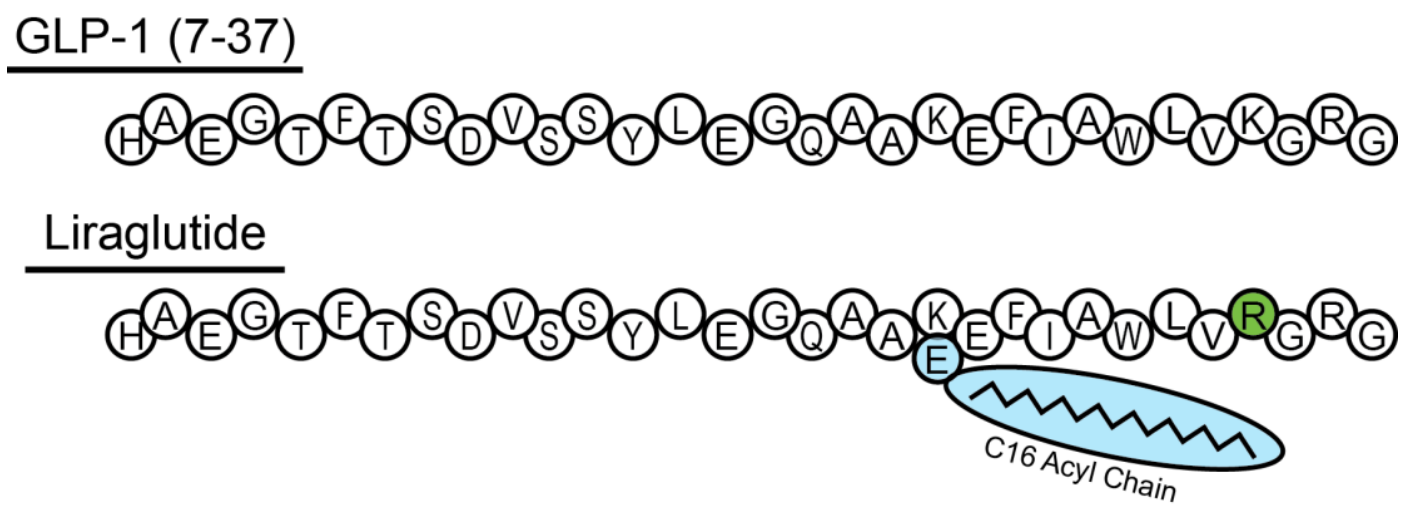

Figure S1. Comparison of Liraglutide to the GLP-1 (7-37) peptide. Highlighted are the sites of conjugation (blue) and amino acid substitution (green).

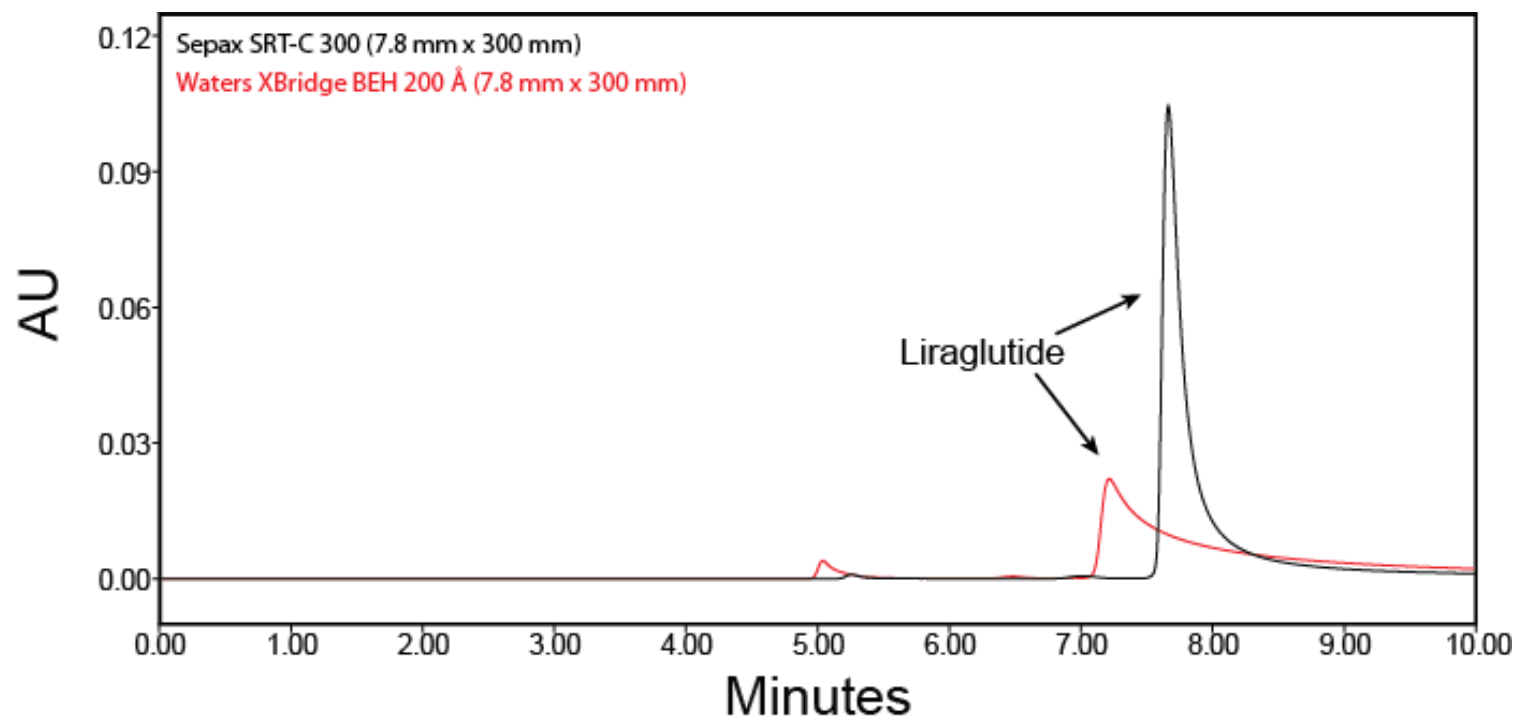

Figure S2. Challenges of SEC method development for conjugated peptides with C16 acyl chain. Example shown is a comparison between a Sepax "C" series column and a Waters Xbridge BEH SEC column. The mobile phase was $10 \mathrm{mM}$ sodium phosphate pH 8.1 with a flow rate of $1.0 \mathrm{~mL} / \mathrm{min}$ and an injection volume of $5 \mu \mathrm{L}$ of $4 \mathrm{mg} / \mathrm{mL}$ Liraglutide $\mathrm{pH}$ 8.1. We have observed similar effects for other acylated peptides where the Sepax colu mn provides good performance with minimal secondary interactions. 

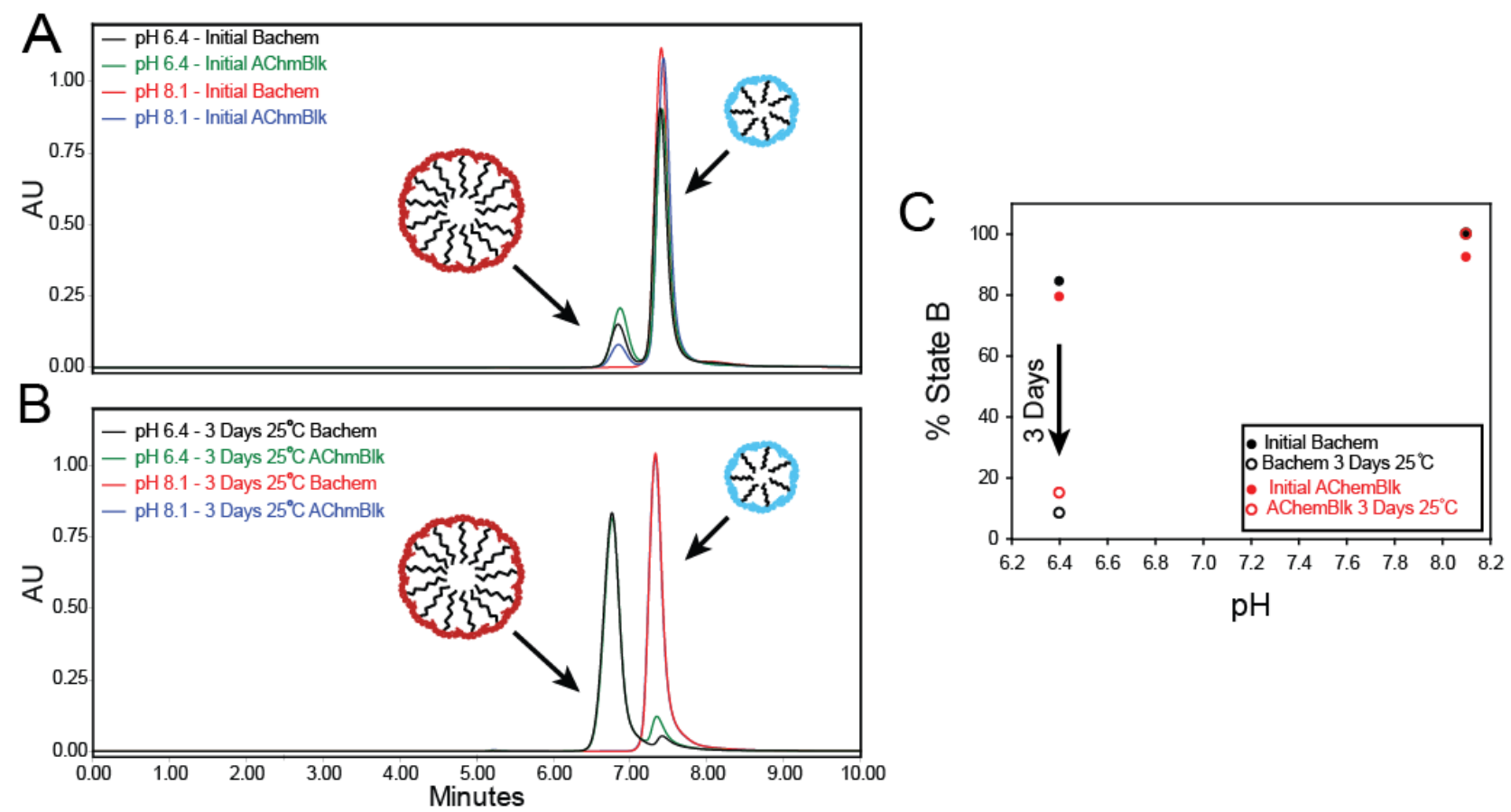

Figure S3. Comparison of synthetic Liraglutide sourced from different vendors Bachem and AChemblock. A) SEC chromatograms of $8 \mathrm{mg} / \mathrm{mL}$ Liraglutide solutions at $\mathrm{pH} 6.4$ and 8.1 recorded immediately after preparation of Liraglutide sourced from Bachem and AChemblock. B) SEC chromatograms of $8 \mathrm{mg} / \mathrm{mL}$ Liraglutide solutions at $\mathrm{pH} 6.4$ and 8.1 recorded after incubation at $25^{\circ} \mathrm{C}$ for 3 days of Liraglutide sourced from Bachem and AChemblock. C) Population of State B determined by SEC peak areas immediately after preparation(filled circles) and after incubation at $25{ }^{\circ} \mathrm{C}$ for 3 days (open circles) of Liraglutide sourced from Bachem (black) and Achemblock (red). 
A

pH 6.4

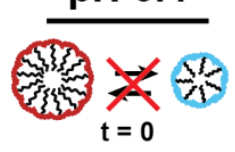

Kinetic

Distribution

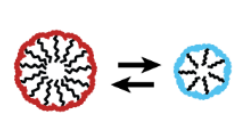

$\mathrm{t}=72 \mathrm{hrs} 25 \mathrm{C}$

Equilibrium

Distribution

B

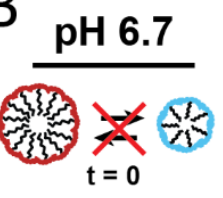

Kinetic

Distribution

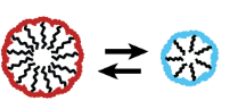

$\mathrm{t}=72 \mathrm{hrs} 25 \mathrm{C}$

Equilibrium

Distribution

\section{C $\mathrm{pH} 7.2$}

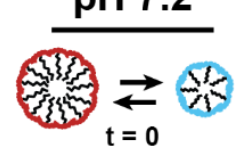

Equilibrium Distribution

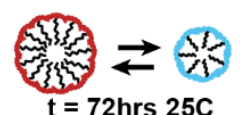

Equilibrium

Distribution

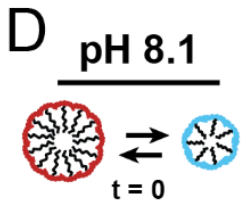

Equilibrium

Distribution

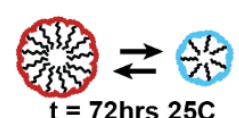

Equilibrium

Distribution
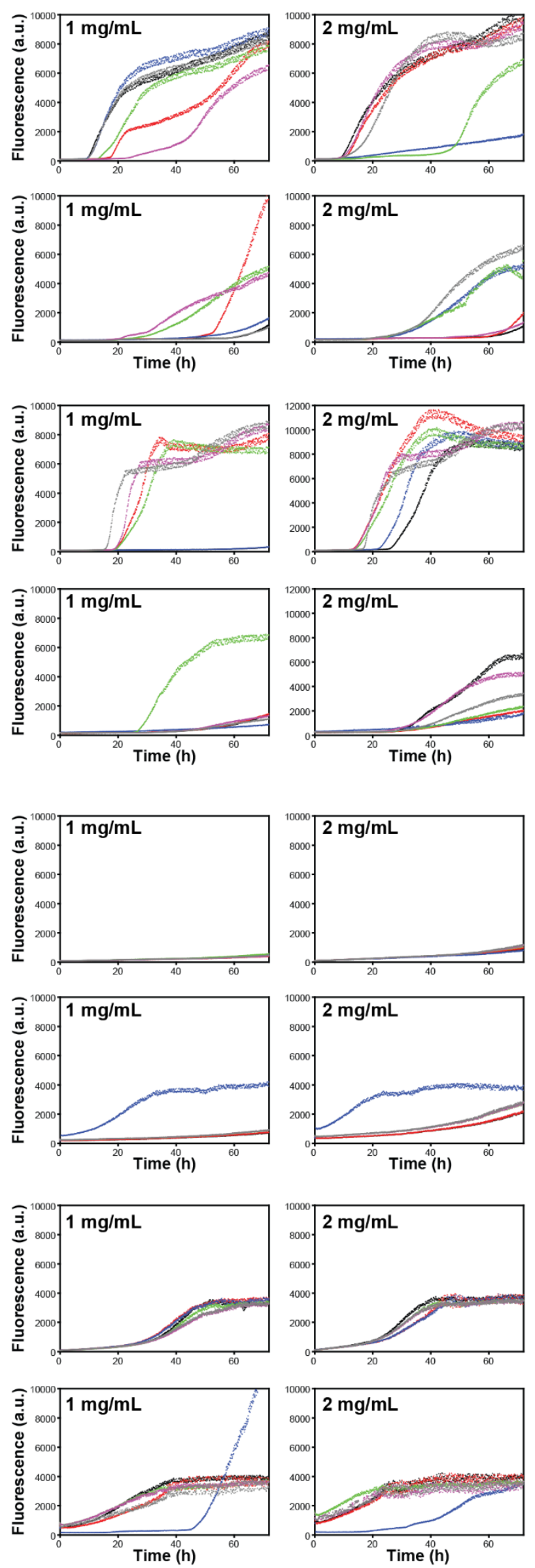
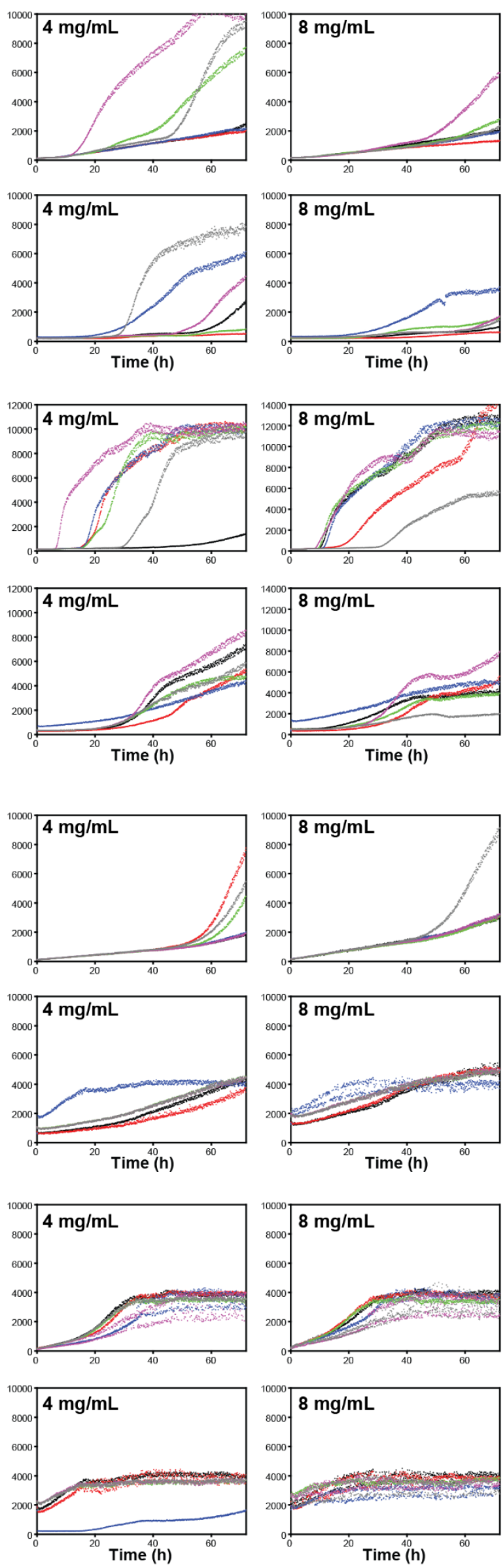
Figure S4. Fibrillation of 1-8 mg/mL Liraglutide solutions upon immediate sample preparation and after incubation at $25^{\circ} \mathrm{C}$ for three days. A: ThioflavinT fluorescence profile recorded during physical stress of Liraglutide solutions at $\mathrm{pH} 6.4$ immediately after preparation(top) and after incubation at $25{ }^{\circ} \mathrm{C}$ for three days (bottom). B: ThioflavinT fluorescence profile recorded during physical stress of Liraglutide solutions at $\mathrm{pH} 6.7 \mathrm{immediately}$ after preparation(top) and after incubation at $25^{\circ} \mathrm{C}$ for three days (botto m). C: Thioflavin T fluorescence profile recorded during physical stress of Liraglutide solutions at $\mathrm{pH} 7.2$ immediately after preparation(top) and after incubation at $25{ }^{\circ} \mathrm{C}$ for three days (bottom). D: ThioflavinT fluorescence profile recorded during physical stress of Liraglutide solutions at $\mathrm{pH} 8.1$ immediately after preparation(top) and after incubation at $25^{\circ} \mathrm{C}$ for three days (bottom). 


\section{pH 6.4 Kinetic}

A
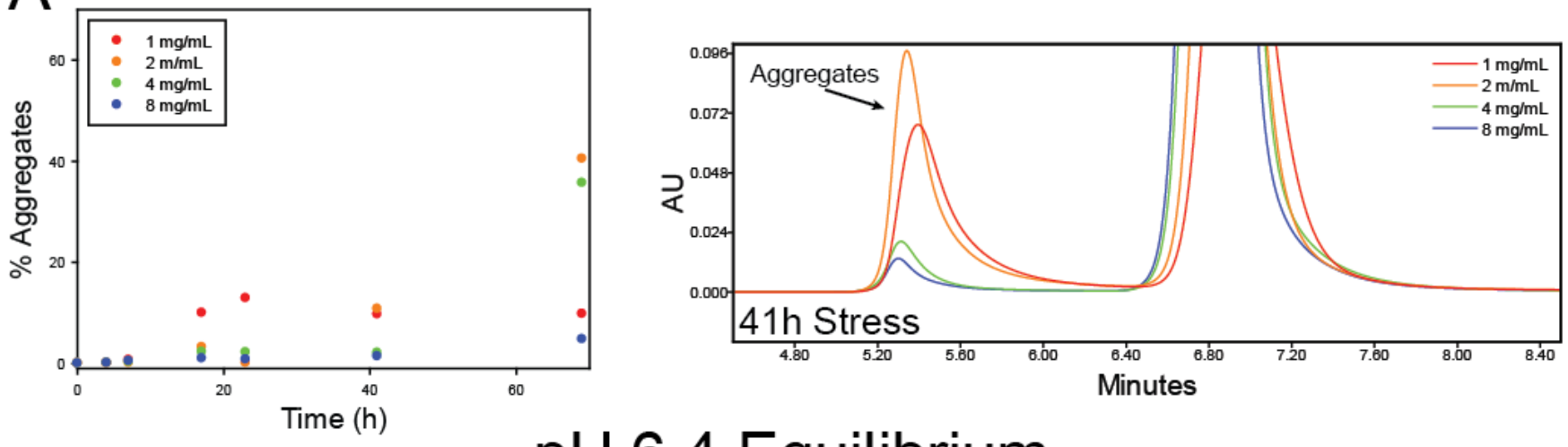

pH 6.4 Equilibrium
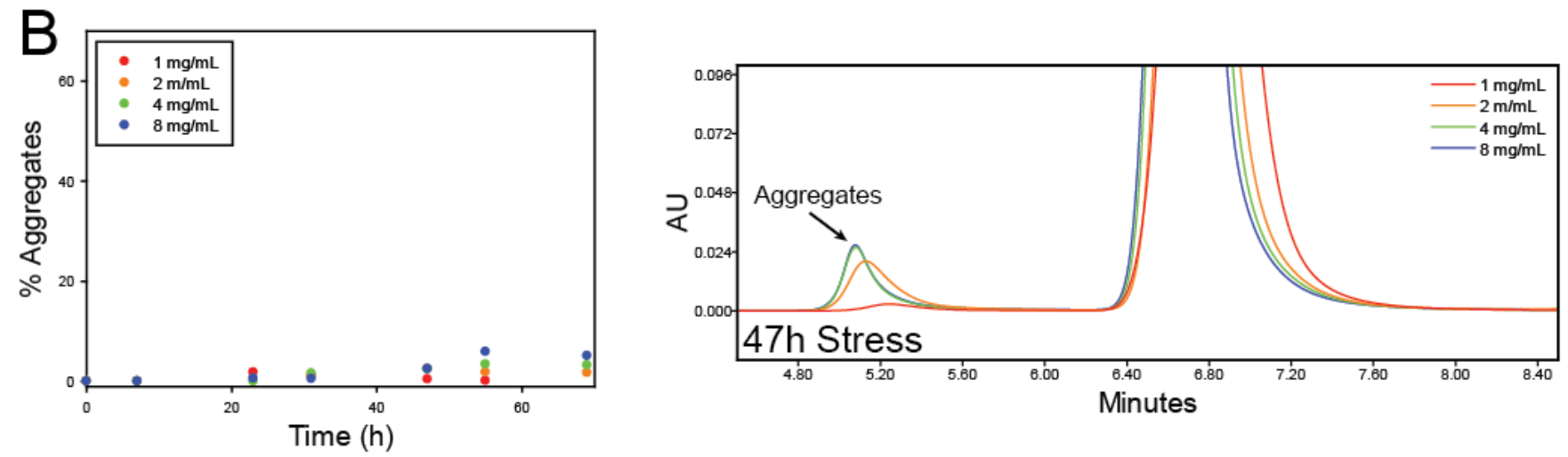

pH 8.1 Equilibrium
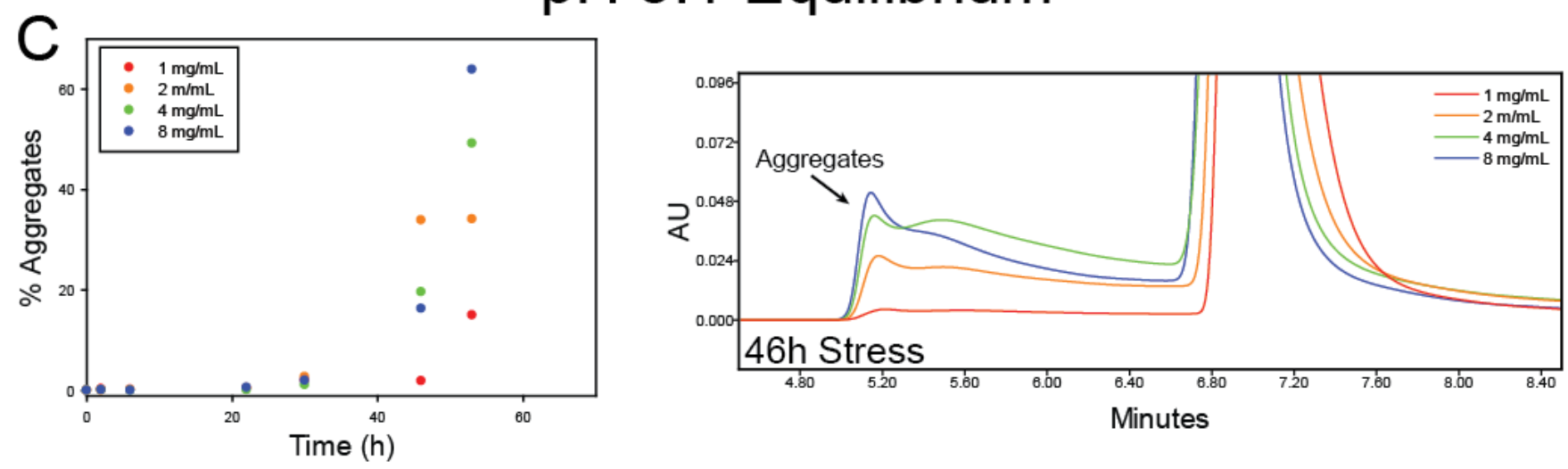

Figure S5. SEC characterization of soluble species collected during thioflavin T physical stress. Samples were collected from the stress plate and injected neat onto the column for SEC-MALS characterization. A) Samples collected from pH 6.4 kinetic equilibrium stress. Left, Percentage of high molecular weight aggregates relative to a reference standard. Right, example SEC chromatograms for samples collected at 41 hours. Note the low level of aggregates at $1 \mathrm{mg} / \mathrm{mL}$ at 69 hours had a corresponding significantly low level of fluorescence in the thioflavin T stress profile. B) Samples collected from pH 6.4 equilibrium stress. Left, Percentage of high molecular weight aggregates relative to a reference standard. Right, example SEC chromatograms for samples collected at 47 hours. C) Samples collected from pH 8.1 equilibrium stress. Left, Percentage of high molecular weight aggregates relative to a reference standard. Right, example SEC chromatograms for samples collected at 46 hours. 

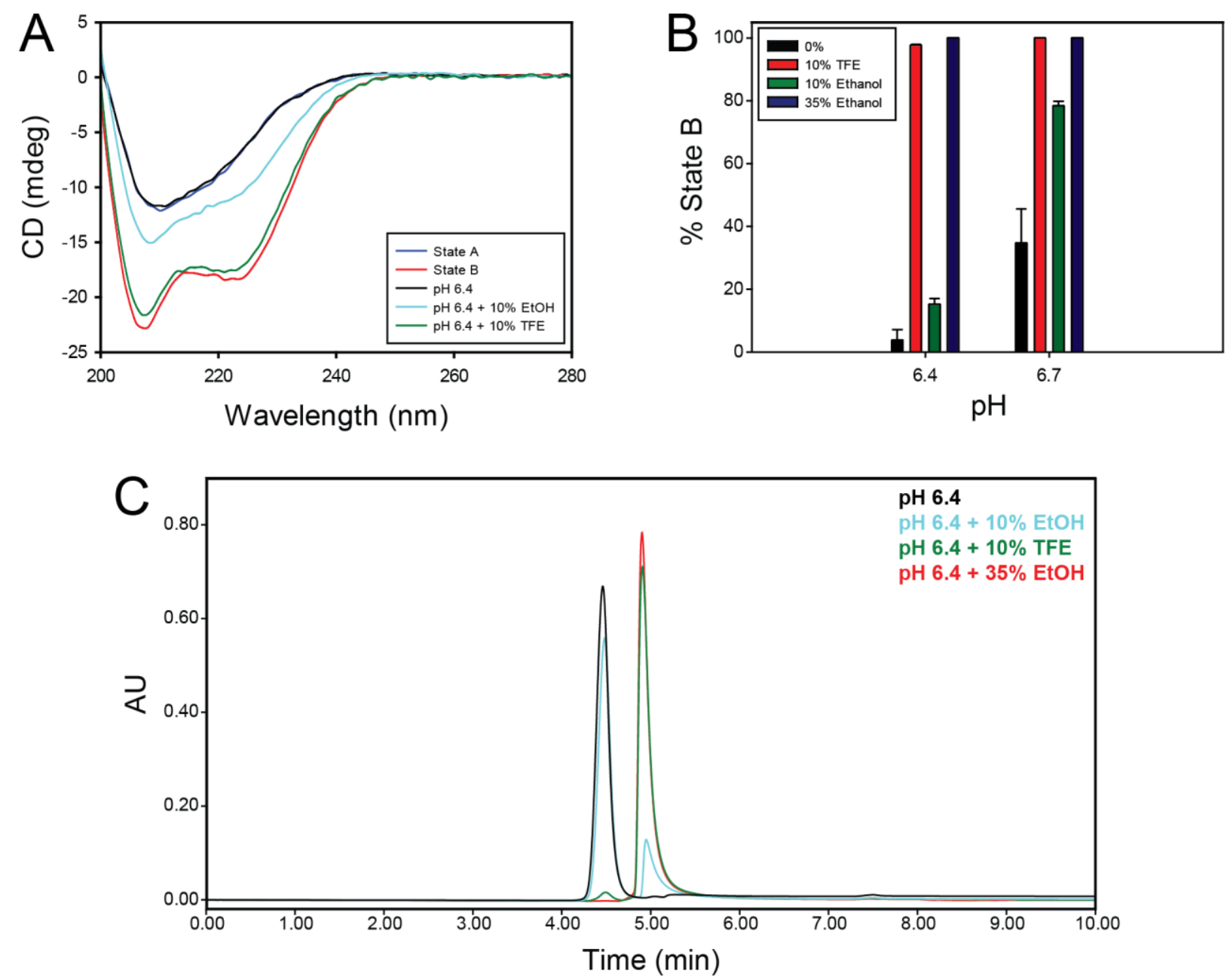

Figure S6. Influence of alcohols on Liraglutide's oligomerization states. A) CD spectra of pH 6.4 Liraglutide solutions incubated with and without TFE and ethanol. Reference spectra for pure States A and B are shown. B) Populations of Liraglutide State B at pH 6.4 and $\mathrm{pH} 6.7$ determined by SEC after incubation of $2 \mathrm{mg} / \mathrm{mL}$ Liraglutide at room temperature with TFE and ethanol (concentrations indicated in key). C) SEC chromatograms of $\mathrm{pH} 6.4$ Liraglutide after incubation with and without TFE and ethanol. 


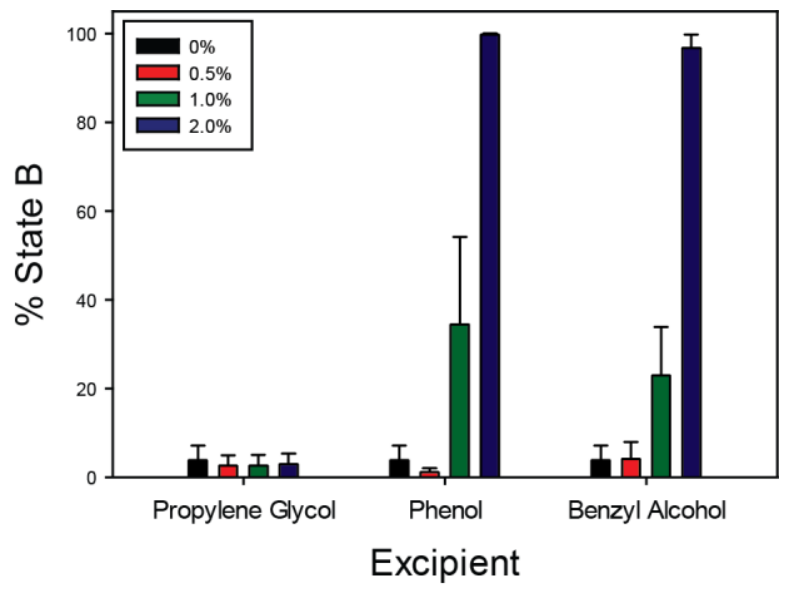

Figure S7. Influence of excipients on Liraglutide's oligomerization states. Shown are the populations of Liraglutide State B at pH 6.4 determined by SEC after storage of $2 \mathrm{mg} / \mathrm{mL}$ Liraglutide for 48 hours at room temperature with varying excipients (concentrations indicated in key). 